Case Report

\title{
Spontaneous Perforation of Common Bile Duct: A Rare Presentation of Gall Stones Disease
}

\author{
Duminda Subasinghe, Edippuli Arachchige Don Udayakumara, \\ Upul Somathilaka, and Milinda Huruggamuwa
}

Department of General Surgery, General Hospital, 60000 Kurunegala, Sri Lanka

Correspondence should be addressed to Duminda Subasinghe; dumindas1982@yahoo.com

Received 15 April 2016; Accepted 6 June 2016

Academic Editor: Engin Altintas

Copyright (C) 2016 Duminda Subasinghe et al. This is an open access article distributed under the Creative Commons Attribution License, which permits unrestricted use, distribution, and reproduction in any medium, provided the original work is properly cited.

\begin{abstract}
Background. Spontaneous perforation of the extrahepatic biliary system is a rare presentation of gall stones. Very few cases of bile duct perforation have been reported in adults. It is rarely suspected or correctly diagnosed preoperatively. Case Presentation. A 66-year-old female presented at the surgical emergency with 3 days' history of severe upper abdominal pain with distension and repeated episodes of vomiting, as she had evidence of generalized peritonitis and underwent an exploratory laparotomy. A single $0.5 \mathrm{~cm} \times 0.5 \mathrm{~cm}$ free perforation was present on the anterolateral surface of the common bile duct at the junction of cystic duct. A cholecystectomy and the CBD exploration were performed. Conclusion. Spontaneous perforation of the extrahepatic bile duct is a rare but important presentation of gall stones in adults. Therefore, awareness of the clinical presentation, expert ultrasound examination, and surgery are important aspects in the management.
\end{abstract}

\section{Introduction}

Spontaneous perforation of the wall of the extrahepatic or intrahepatic bile duct with biliary peritonitis is a rare event in infants and an extremely rare event in adults. It was first described in 1882 by Freeland [1]. Generalized biliary peritonitis following perforation of the biliary tract is commonly a sequel of rupture of an inflamed gangrenous gall bladder [2]. Bile duct perforation is relatively common in infants $[3,4]$ and is related to congenital biliary system anomalies. The aetiology of biliary tract perforation in the adults is commonly attributable to intramural infection, necrosis of the wall of the bile duct secondary to thrombosis, increased intraductal pressure secondary to obstruction, cirrhosis, and direct erosion by calculi.

The presentation of biliary peritonitis varies and because of its rarity the correct preoperative diagnosis is often difficult and delayed. This along with the associated comorbidity of mostly the elderly, patients in whom it is seen, results in a mortality rate of $30-50 \%$ [4]. We report an interesting, rare case of spontaneous extrahepatic bile duct perforations with choledocholithiasis, in an adult female. Its clinical presentations, investigative findings, and management are discussed and relevant literatures are reviewed. The rarities of this case are the atypical site of CBD perforation and its occurrence at a relatively older age.

\section{Case Presentation}

A 66-year-old female presented at the surgical emergency with 3 days' history of severe upper abdominal pain with distension and repeated episodes of vomiting. The abdominal pain increased in intensity and became continuous and more diffuse for the last two days. Her past medical, surgical, and family histories were unremarkable. General examination revealed that she was dehydrated, febrile $(101 \mathrm{~F})$, and having a pulse rate of 120/minute and blood pressure of $90 / 70 \mathrm{~mm}$ of $\mathrm{Hg}$. She was not icteric and free of cervical lymphadenopathy. Her abdomen was distended with guarding and rebound tenderness mainly in the upper abdomen. There was no evidence of free fluid in the abdomen. Liver dullness was not obliterated. Bowel sounds were absent.

Hematological investigations revealed neutrophil leukocytosis $\left[17700\right.$ cells $/ \mathrm{mm}^{3}$ (84\% neutrophil)] with normal 


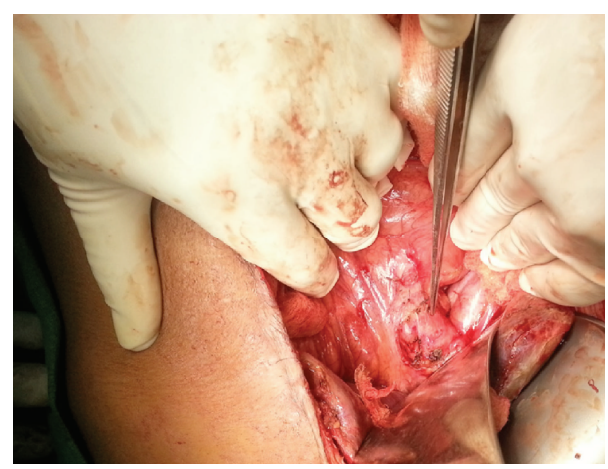

Figure 1: Perforation on the anterolateral surface of the common bile duct.

total bilirubin [10.2 $\mu \mathrm{mol} / \mathrm{L}$ (5-21)], alkaline phosphatase [255 IU/L (70-360)], and alanine transaminase [14 IU/L (8$38 \mathrm{IU} / \mathrm{L})]$. Serum amylase level was normal. Her erect chest radiograph was normal without evidence of pneumoperitoneum but on USS abdomen showed small amount of free fluid in hepatorenal pouch. Therefore, definite preoperative diagnosis was not possible. As she had evidence of generalized peritonitis, she underwent an exploratory laparotomy, after adequate resuscitation and under broad spectrum antibiotic coverage.

Operative findings included bilious peritoneal fluid in dependent parts of the peritoneal cavity with localized collection of bile inside the lesser sac. Gall bladder was distended with thickened wall and contained multiple stones. A single $0.5 \mathrm{~cm} \times 0.5 \mathrm{~cm}$ free perforation was present on the anterolateral surface of the common bile duct at the junction of cystic duct (Figure 1). The common bill duct was dilated and contained multiple stones. A cholecystectomy was done and the CBD was explored. Multiple small stones were removed, irrigation was done with warm normal saline, and the CBD was closed over a T-tube inserted through the site of perforation. The peritoneal cavity was thoroughly irrigated with warm normal saline. The abdomen was closed after inserting a suction drain in the hepatorenal space. Unhealthy edges of the perforation site were excised and sent for the histology which subsequently showed inflamed necrotic tissue without any evidence of malignant cells. The histology of the gallbladder was suggestive of chronic cholecystitis.

The patient made a slow postoperative recovery. There was persistent drainage of bile through the subhepatic drain until the 10th postoperative day. T-tube cholangiogram performed on the 10th postoperative day showed leaking and the pooling of contrast at T-tube insertion site of CBD with leaking tract extending towards drain site (Figure 2). It also showed that there was a filling defect in the distal CBD with contrast flow into the duodenum. Therefore, subsequently she underwent endoscopic retrograde cholangiopancreatography and extraction of distal CBD stone. T-tube was removed on the 30th postoperative day and she was discharged on 35th postoperative day. She was doing well at the time of the first visit and on follow-up visits until 1 year.

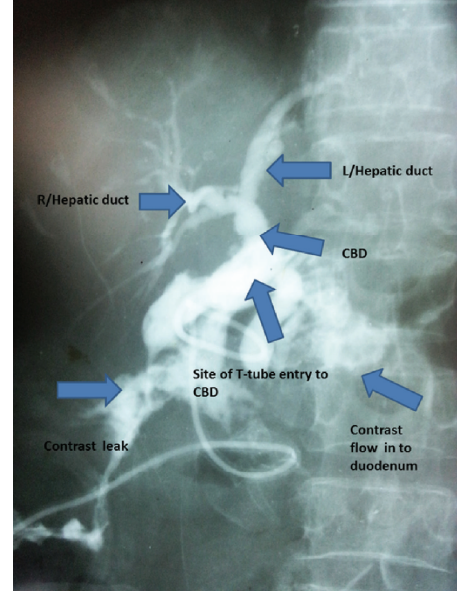

Figure 2: Postoperative T-tube cholangiogram showing biliary anatomy and contrast leak.

\section{Discussion}

Spontaneous perforation of common bile duct is a rare cause of biliary peritonitis. It is rarely suspected or diagnosed preoperatively. Perforation of the biliary system is a recognized complication of cholelithiasis; the diagnosis should be suspected if a perihepatic abscess or peritonitis is combined with biliary stone disease. Kang et al. [5] reviewed 70 cases of spontaneous bile duct perforations in adults. Among these, 42 patients had perforation in common bile duct, followed by hepatic duct in 28 cases. Although the pathogenesis of spontaneous biliary perforation is poorly understood, the commonest cause for perforation was a stone in adults. The recognized mechanisms include calculus perforation at the site of impaction and erosion. The other causes for spontaneous perforation of bile duct reported in literature were increased canalicular pressure due to obstruction by tumour, spasm of the sphincter of Oddi, intramural infection, mural vessel infarction leading to mural necrosis, or rupture of a biliary tract anomaly such as cyst or biliary diverticulum, a connective tissue defect, or ischemic compromise that results in perforation of the duct wall and previous biliary tract surgeries [6-9]. Sometimes it can be idiopathic. Talwar et al. [10] have reported a case of $\mathrm{CBD}$ perforation during pregnancy and the site of the perforation was supraduodenal portion of the CBD with stone impaction. Our patient's ultrasound abdomen showed perihepatic fluid collection suggesting a diagnosis of perforated viscus but there gall stones were not visualized. It is important to mention that ultrasound scan of the abdomen is an operator dependent imaging modality. Therefore, our patient's preoperative diagnosis was uncertain and therefore the definitive diagnosis was made intraoperatively.

Perforation of the common bile duct was most probably related to the abrupt increase in local intraluminal pressure causing erosion of the stones. This results in abrupt increase in intraluminal pressure and decreased blood flow in the vessels which run along the lateral border of the bile ducts, resulting in ischemia on the anterior surface of the bile duct 
[6]. This might explain the site of CBD perforation in our patient. The commonest reported site of perforation is at the junction of the cystic and hepatic ducts $[8,11]$ and our patient's findings were compatible with previous literature. This patient presented with a 3-day delay with generalized peritonitis. Facilities for the intraoperative cholangiogram or ERCP were not available. Recommended treatment in this situation is T-tube drainage of the common bile duct along with cholecystectomy. In cases with distal obstruction of the CBD, a biliary enteric bypass should be done. Primary suture repair of the $\mathrm{CBD}$ is considered if a preoperative cholangiogram is available and it shows no pathology distal to the perforation. Therefore, in the absence of intraoperative cholangiogram, this patient's condition was best managed by closure over a T-tube [12] followed by postoperative ERCP in a specialized hepatobiliary center [12]. The mainstay of the management of patients with suspected stones in the common bile duct has three aims: to evaluate the probability of stones in the common bile duct, to treat these stones when present, and to treat the source of the stones, gallbladder. The most common treatment modality is ERCP, with duct cannulation and clearance rates reaching $98 \%$ in expert hands [13]. Laparoscopic common bile duct exploration and clearance of stones were found to be as effective as preoperative and postoperative ERCP, with no significant difference in morbidity and mortality [14].

\section{Conclusion}

In conclusion, spontaneous perforation of the extrahepatic bile duct is a rare but important presentation of gall stones but important condition in adults. Therefore, awareness of the clinical presentation and expert ultrasound examination are important adjuncts in the diagnosis. Conservative surgery is the mainstay of treatment in the acute presentation.

\section{Abbreviations}

CBD: Common bile duct

USS: Ultrasound scan

ERCP: Endoscopic retrograde cholangiopancreatography.

\section{Consent}

Written informed consent was obtained from the patient for publication of this case report and accompanying images.

\section{Competing Interests}

The authors declare no competing interests regarding the publication of this paper.

\section{Authors' Contributions}

Duminda Subasinghe participated in the conception and design of the report and wrote the paper and Edippuli Arachchige Don Udayakumara analyzed the report. Edippuli Arachchige Don Udayakumara, Duminda Subasinghe, Upul
Somathilaka, and Milinda Huruggamuwa have been involved in the diagnosis, surgical management, and follow-up of the patient. All authors read and approved the final paper. All four authors were involved in planning, analysis of the case, and writing of the paper.

\section{Acknowledgments}

The authors would like to thank the ward staff of the hospital for providing support and helping in management of the patient.

\section{References}

[1] J. Freeland, "Rupture of the hepatic duct," The Lancet, vol. 119, no. 3062, pp. 731-732, 1882.

[2] R. Lochan and B. V. Joypaul, "Bile peritonitis due to intrahepatic bile duct rupture," World Journal of Gastroenterology, vol. 11, no. 42, pp. 6728-6729, 2005.

[3] C. Chardot, F. Iskandarani, O. De Dreuzy et al., "Spontaneous perforation of the biliary tract in infancy: a series of 11 cases," European Journal of Pediatric Surgery, vol. 6, no. 6, pp. 341-346, 1996.

[4] S. Marwah, J. Sen, A. Goyal, N. Marwah, and J. P. Sharma, "Spontaneous perforation of the common bile duct in an adult," Annals of Saudi Medicine, vol. 25, no. 1, pp. 58-59, 2005.

[5] S.-B. Kang, H.-S. Han, S. K. Min, and H. K. Lee, "Nontraumatic perforation of the bile duct in adults," Archives of Surgery, vol. 139, no. 10, pp. 1083-1087, 2004.

[6] K. Ando, T. Miyano, S. Kohno, S. Takamizawa, and G. Lane, "Spontaneous perforation of choledochal cyst: a study of 13 cases," European Journal of Pediatric Surgery, vol. 8, no. 1, pp. 23-25, 1998.

[7] L. J. Gariepy, O. A. Capano, and L. W. Gardner, "Non-traumatic rupture of the common bile duct," The American Journal of Surgery, vol. 81, no. 3, pp. 357-362, 1951.

[8] T. Hasegawa, Y. Udatsu, M. Kamiyama et al., "Does pancreaticobiliary maljunction play a role in spontaneous perforation of the bile duct in children?" Pediatric Surgery International, vol. 16, no. 8, pp. 550-553, 2000.

[9] N. G. Steinhoff and G. L. Tucker, "Nontraumatic perforation of the common duct," The American Journal of Surgery, vol. 121, no. 3, pp. 334-337, 1971.

[10] N. Talwar, M. Andley, B. Ravi, and A. Kumar, "Spontaneous biliary tract perforations: an unusual cause of peritonitis in pregnancy. Report of two cases and review of literature," World Journal of Emergency Surgery, vol. 1, no. 1, article 21, 2006.

[11] G. Stringel and S. Mercer, "Idiopathic perforation of the biliary tract in infancy," Journal of Pediatric Surgery, vol. 18, no. 5, pp. 546-550, 1983.

[12] N. Spigland, R. Greco, and D. Rosenfeld, "Spontaneous biliary perforation: does external drainage constitute adequate therapy?" Journal of Pediatric Surgery, vol. 31, no. 6, pp. 782-784, 1996.

[13] B. Schumacher, T. Frieling, D. Haussinger, and C. Niederau, "Endoscopic treatment of symptomatic choledocholithiasis," Hepato-Gastroenterology, vol. 45, no. 21, pp. 672-676, 1998.

[14] D. J. Martin, D. R. Vernon, and J. Toouli, "Surgical versus endoscopic treatment of bile duct stones," Cochrane Database of Systematic Reviews, no. 2, Article ID CD003327, 2006. 


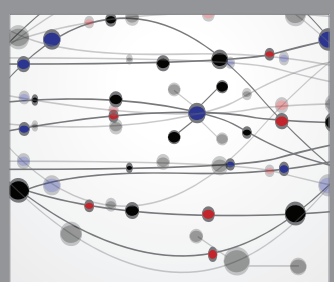

The Scientific World Journal
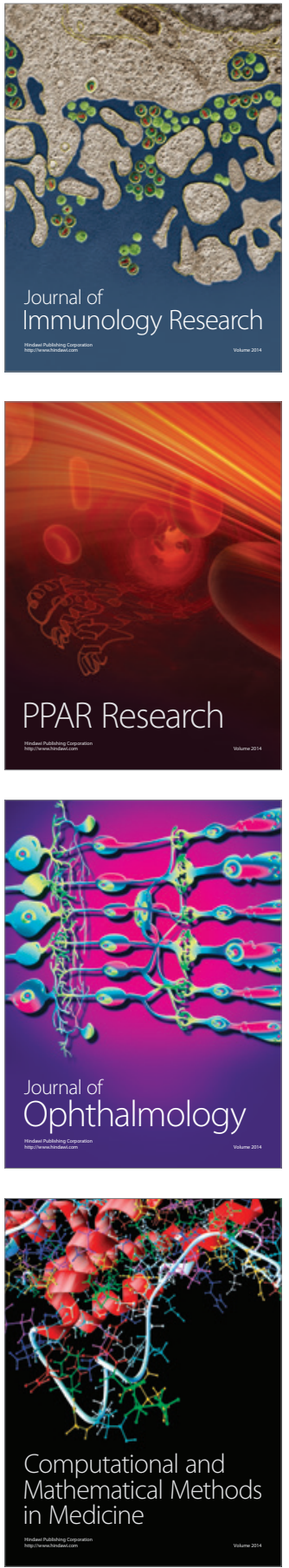

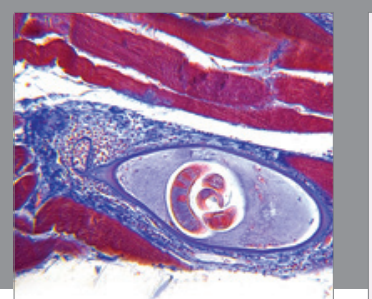

Gastroenterology Research and Practice

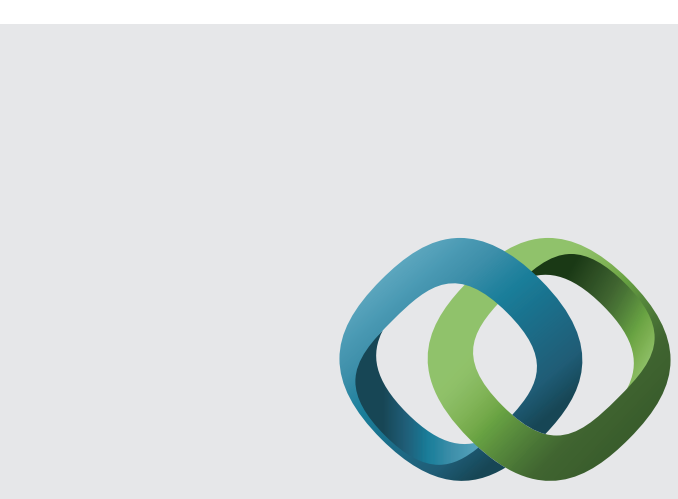

\section{Hindawi}

Submit your manuscripts at

http://www.hindawi.com
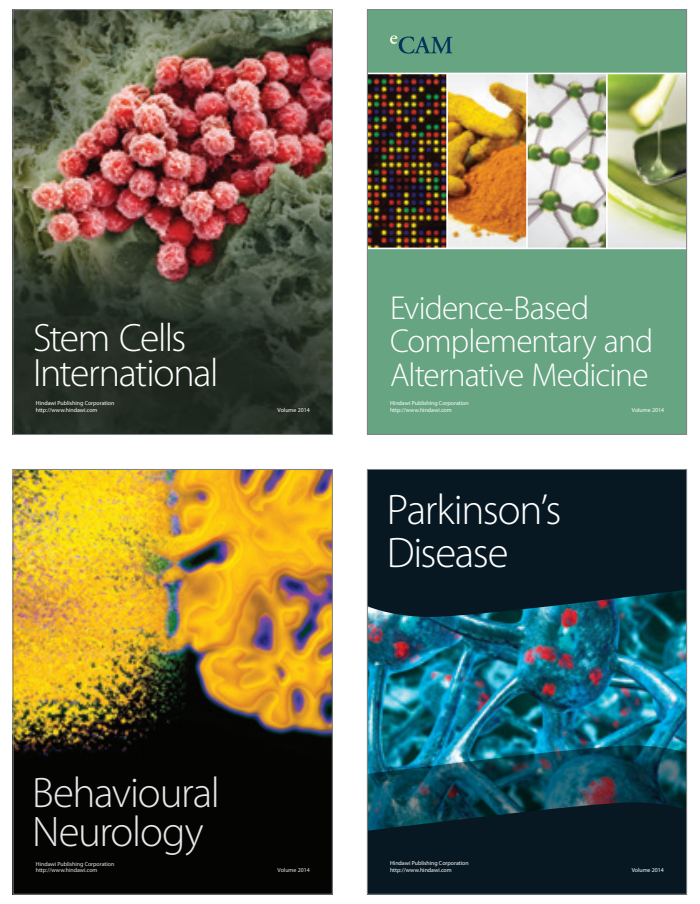
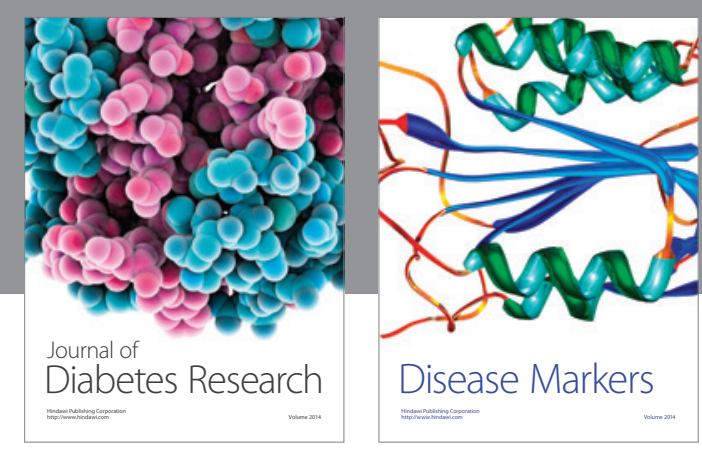

Disease Markers
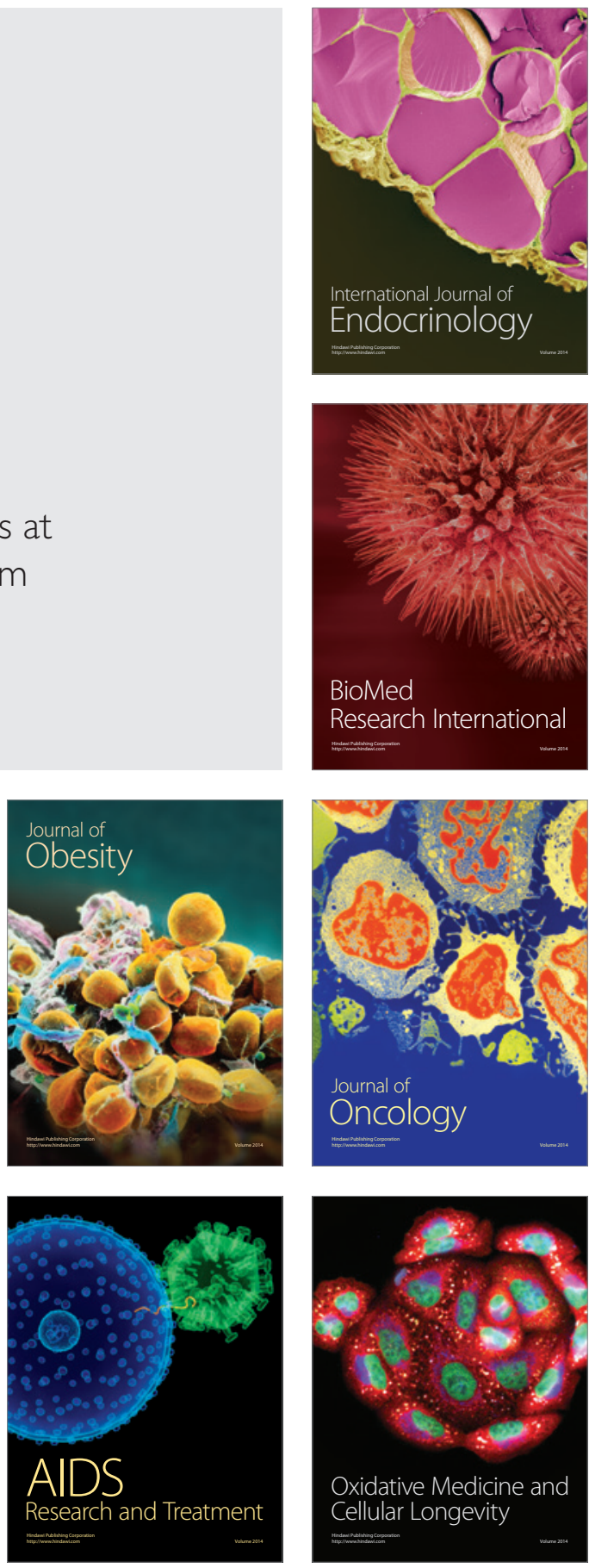